Research Article

\title{
Effect of Omega-3 Fatty Acid Alone and in Combination with Proprietary Chromium Complex on Endothelial Function in Subjects with Metabolic Syndrome: A Randomized, Double-Blind, Parallel-Group Clinical Study
}

\author{
Usharani Pingali $(\mathbb{D}$, Chandrasekhar Nutalapati, and Srinivas Gundagani \\ Department of Clinical Pharmacology \& Therapeutics, Nizam's Institute of Medical Sciences, Panjagutta, Hyderabad 500082, \\ Telangana, India \\ Correspondence should be addressed to Usharani Pingali; ushapingali@yahoo.com
}

Received 18 July 2020; Revised 1 February 2021; Accepted 12 June 2021; Published 25 June 2021

Academic Editor: Eun Jung Sohn

Copyright (c) 2021 Usharani Pingali et al. This is an open access article distributed under the Creative Commons Attribution License, which permits unrestricted use, distribution, and reproduction in any medium, provided the original work is properly cited.

\begin{abstract}
Metabolic syndrome (MetS) represents a cluster of metabolic abnormalities that include hypertension, central obesity, insulin resistance, and dyslipidemia and is strongly associated with an increased risk of diabetes, cardiovascular diseases (CVD), and allcause mortality. Early diagnosis is important to employ lifestyle and risk factor modification. Existing therapies are limited. Studies report positive effect of omega-3 fatty acids ( $\omega$-3FA) on symptoms of metabolic syndrome. The present study was undertaken to evaluate the effect of $\omega$-3FA alone and in combination with proprietary chromium complex (PCC) on endothelial function in subjects with metabolic syndrome. In this randomized, double-blind, parallel-group study, subjects were enrolled into the study after ethics committee (EC) approval and informed consent. Eligible subjects were randomized to receive $\omega$-3FA concentrate $2000 \mathrm{mg}$ (Group A-18 subjects), $\omega$-3FA concentrate $2000 \mathrm{mg}+$ PCC200 mcg (Group B-19 subjects), and $\omega$-3FA concentrate $2000 \mathrm{mg}+$ PCC400 mcg (Group C-21 subjects) daily for 12 weeks. Endothelial dysfunction as measured by reflection index (RI), biomarkers of oxidative stress (NO, MDA, and glutathione), and inflammation (hsCRP, endothelin-1, ICAM-1, and VCAM-1) were evaluated at baseline, 4, and 12 weeks. Lipid-profile and platelet-aggregation tests were performed at baseline and 12 weeks. Adverse drug reactions were recorded. Compliance was assessed by pill count method. GraphPad Prism 8 was used for statistical analysis. Significant changes were seen from 4 weeks onwards in all the parameters evaluated. Significant improvement in $\mathrm{RI} \%$ (mean $\pm \mathrm{SD}=-2.56 \pm 0.77$ to $-3.27 \pm 0.67$-group $\mathrm{A},-2.33 \pm 0.76$ to $4.72 \pm 0.79$-group $\mathrm{B} ;-2.39 \pm 1.13$ to $6.46 \pm 1.00$-group C) was seen at 12 weeks. Significant improvement in biomarkers of oxidative stress and inflammation was seen with all the treatment groups. Similarly, significant improvement in lipid profile was seen in group B and group C, while group A showed change in HDL, VLDL, and TG. Group C demonstrated the best response in the parameters evaluated. Three patients in group C reported gastrointestinal adverse events, which resolved spontaneously; none stopped the therapy. So, the addition of PCC to $\omega$-3FA may prove to have beneficial effect in reducing cardiovascular morbidity in MetS patients.
\end{abstract}

\section{Introduction}

Metabolic syndrome (MetS) is defined by a constellation of interconnected physiological, biochemical, clinical, and metabolic factors that directly increases the risk of cardiovascular disease, type 2 diabetes mellitus, and all-cause mortality $[1,2]$. Insulin resistance, visceral adiposity, atherogenic dyslipidemia, endothelial dysfunction, genetic susceptibility, elevated blood pressure, hypercoagulable state, and chronic stress are the several factors that constitute the syndrome [3]. It is a significant and escalating public health and clinical challenge worldwide in the wake of urbanization, surplus energy intake, increasing obesity, and sedentary life habits. MetS increases the risk of T2DM, CVD 
[4], stroke and MI, and dying from such event [5] regardless of a previous history of cardiovascular events [6].

A strong association between MetS diagnosed according to the Adult Treatment Panel (ATP) III criteria [7] and oxidative stress has been found. Hyperglycemia and inflammation, important components of MetS, increase the production of reactive oxygen species (ROS) [8], resulting in increased oxidative stress with overactivation of NADPH oxidase. This process reduces the bioavailability of nitric oxide (NO) [9], explaining both the low NO levels in subjects with metabolic disorders and the correlation of NO levels with body mass index (BMI), blood pressure, and triglyceridemia [10]. Thus, decreased NO availability appears to play a significant role in MetS. Glutathione (GSH) is one of the essential antioxidant mechanisms in living organisms. Low GSH levels are associated with oxidative stress and the manifestation of various diseases [11]. Similarly, malondialdehyde (MDA) is a biomarker of intensified lipid peroxidation, which leads to the production of free radicals. Studies have shown that increased oxidative stress through lipid peroxidation may lead to diabetes [12].

Studies have shown that population with MetS have increased levels of inflammatory markers like hsCRP. There also seems to be an association of hsCRP with adhesion molecules such as intercellular adhesion molecule-1 (ICAM1) and vascular cell adhesion molecule-1 (VCAM-1) [13]. Circulating levels of both soluble ICAM-1 and VCAM-1 have been reported in MetS. The levels of soluble adhesion molecules like sICAM-1 and sVCAM-1 reflect the expression of membrane-bound adhesion molecules and the process of vascular inflammation of the vessel wall [14]. Similarly, endothelin-1 (ET-1), which is linked to vascular inflammation, may also play an essential role in the development of arteriosclerosis along with adhesion molecules. It also seems to have vasoconstrictor activity. So, increased production or activity of endothelin-1 may lead to MetS [15]. Data on such biomarkers in subjects with MetS is scanty.

Studies report that excess cholesterol substantially alters the endothelial function, leading to a decreased relaxation of the arterial vessels $[16,17]$. The defect has been described mainly in atherosclerotic subjects, as well as in asymptomatic subjects with hypercholesterolemia [18]. Data from the MRFIT study [19] show that the relationship between cholesterol and coronary artery disease is active and graded across all levels of blood pressure; similarly, the relationship between blood pressure and coronary artery disease is substantial and graded across all levels of cholesterol.

Despite advancement in our understanding of metabolic syndrome currently, there is no single therapy approved to manage it as a single condition, as it is a multifaceted health problem. The mainstay of treatment includes a healthy lifestyle, which may include promoting physical activity for weight reduction and a balanced diet. These are likely to be the most effective in controlling metabolic syndrome; nonetheless, it is challenging to initiate and maintain a healthy lifestyle. Pharmacotherapy may include agents that deal with different components of MetS like obesity, diabetes, hypertension, and dyslipidemia that are used singly or in combination: antiobesity drugs, thiazolidinediones, metformin, statins, fibrates, renin-angiotensin system blockers, glucagon-like peptide-1 agonists, sodium-glucose transporter-2 inhibitors, and some antiplatelet agents such as cilostazol. Nevertheless, multidrug treatment may lead to drug interactions, decreases patient compliance, and increases health costs, so it becomes relevant to introduce single therapies for treating different aspects of MetS. In such a scenario, nutraceutical may have an important role to play [20-23].

Omega-3 polyunsaturated fatty acids (PUFAs), such as eicosapentaenoic acid and docosahexaenoic acid, are widely regarded as cardioprotective. Large-scale, randomized clinical trials have reported that dietary intake of omega-3 PUFAs improves the prognosis of patients with symptomatic heart failure or recent myocardial infarction [24]. Animal studies have shown that omega- 3 fatty acid may possess potent antioxidant and anti-inflammatory properties and may prevent fibrosis of different organs [25]. It also empowers the antioxidant defense system by reducing oxidative stress and increasing the activities of the major antioxidant enzymes such as superoxide dismutase, catalase, and glutathione peroxidase [26]. The American Heart Association recommends that all adults eat fish 2 to 3 times per week because of its content of $\omega$-3FA. $\omega$-3FA has been shown to have positive cardiovascular effects including lowering triglycerides, blood pressure, and platelet aggregation, increasing arterial compliance and improving endothelial function [27].

Previous studies done at our department had shown that a proprietary chromium complex (PCC) improved endothelial function, biomarkers of oxidative stress, and lipid profile significantly [28]. PCC is prepared by complexation of $\mathrm{Cr}^{3+}$ chloride with an aqueous extract of Phyllanthus emblica fruits, which contain polyphenolic compounds, and purified Shilajit, a herbomineral product, which, by virtue of its liposomal fulvic acid content, increases the bioavailability of chromium. PCC has been shown to have synergistic activity to improve endothelial function and cardiovascular health (US Patent 10,183,047). Studies report that Shilajit induces the growth of blood vessels [29] and has a prominent cardioprotective effect [30]. In a study done by us [31], we had evaluated the effect of fish oil alone and in combination with different doses of proprietary chromium complex (PCC) in type 2 diabetes patients. We had demonstrated that fish oil, in combination with PCC, significantly improved endothelial function. The present prospective, randomized, double blind, parallel group study was, thus, undertaken to evaluate the effect of combining $\omega$-3FA alone and with two doses of PCC (10 mg \& $20 \mathrm{mg})$ versus $\omega$-3FA alone on endothelial function, systemic inflammation, vascular inflammation, and lipid profile in subjects with metabolic syndrome. Furthermore, the study was designed to evaluate study medications in improving the various parameters of metabolic syndrome.

\section{Materials and Methods}

This 12-week prospective, randomized, double-blind, parallel-group study was conducted in the Department of 
Clinical Pharmacology and Therapeutics at NIMS, Hyderabad, India, between the period from November 2017 to June 2018.

2.1. Enrollment of Study Subjects. The subjects were recruited from the Outpatient Department of General Medicine. The participants were provided with the subject information sheet, informed consent form, and contact information for study enrollment.

2.1.1. Inclusion Criteria. These include subjects of either sex aged 30-65 years having endothelial dysfunction defined as $\leq 6 \%$ change in reflection index (RI) on postsalbutamol challenge test and those complying with "The International Diabetes Federation" guidelines, dated 2017 [32], which states central obesity (defined as waist circumference with ethnicity-specific values - if BMI is $>30 \mathrm{~kg} / \mathrm{m}^{2}$, central obesity can be assumed, and waist circumference does not need to be measured), and any two of the following: raised triglycerides $>150 \mathrm{mg} / \mathrm{dL}(1.7 \mathrm{mmol} / \mathrm{L})$ or specific treatment for this lipid abnormality, reduced HDL cholesterol: $<40 \mathrm{mg} / \mathrm{dL}(1.03 \mathrm{mmol} / \mathrm{L})$ in males, $<50 \mathrm{mg} / \mathrm{dL}(1.29 \mathrm{mmol} /$ $\mathrm{L})$ in females, or specific treatment for this lipid abnormality, raised blood pressure: systolic $\mathrm{BP}>130$ or diastolic $\mathrm{BP}$ $>85 \mathrm{mmHg}$, or treatment of previously diagnosed hypertension, raised fasting plasma glucose of $\geq 100 \mathrm{mg} / \mathrm{dL}$, previously diagnosed type 2 diabetes were included into the study.

2.1.2. Exclusion Criteria. These include subjects with abnormal hematological, or biochemical parameters considered significant by investigator, uncontrolled hypertension, hypertriglyceridemia, impaired hepatic or renal functions, history of malignancy or stroke, history of smoking/alcoholism, any other severe disease requiring active treatment, and on any other dietary or herbal supplements.

2.2. Ethical Consideration. The present study was approved by Nizam's Institute of Medical Sciences-Institutional Ethics Committee. Written informed consent was taken from all the subjects before participation in the study. This trial was conducted in accordance with the Declaration of Helsinki [33]. Figure 1 depicts Consolidated Standards of reporting Trials (CONSORT) flow chart. This study was registered with Clinical Trials Registry, India (CTRI) with the registration number of https:/CTRI/2018/05/013548; date: 01/05/2018. Retrospectively registered. http://ctri.nic.in/Clinicaltrials/rmaindet.php?trialid $=23364 \&$ EncHid $=82660.16614 \& \operatorname{modid}=1 \&$ compid $=19$.

\subsection{Investigational Products}

2.3.1. Product 1. Each soft gelatin capsule of Product 1 contains $1000 \mathrm{mg}$ of a refined $\omega-3 \mathrm{FA}$, having $300 \mathrm{mg}$ of EPA
(TG) and $200 \mathrm{mg}$ of DHA (TG), and $10 \mathrm{mg}$ of microcrystalline cellulose (excipient).

2.3.2. Product 2. Each soft gelatin capsule of Product 2 contains $1000 \mathrm{mg}$ of a refined $\omega-3 \mathrm{FA}$, containing $300 \mathrm{mg}$ of EPA (TG) and $200 \mathrm{mg}$ of DHA (TG), and $10 \mathrm{mg}$ of PCC, also known as "Crominex ${ }^{\circledR}$," (equivalent to $200 \mathrm{mcg}^{\circ} \mathrm{Cr}^{3+}$ ).

Both products were made at Tishcon Corp., NY, and supplied by Natreon, NJ.

2.4. Randomization and Blinding. A total of 73 subjects were screened out, of which 60 subjects meeting the inclusion criteria were enrolled in the study. They were randomized into one of the three treatment groups in a double-blind fashion using computer-generated block randomization. Two subjects dropped out of the study before the first followup, citing logistical reasons. A total of 58 subjects completed the study.

All the capsules given to the subjects were of similar size, color, shape, and texture. Each subject was given a sealed packet, which was sequentially designated numbers and containing two sealed bottles, each bottle containing 33 capsules. The packets were dispensed by the pharmacist to the subjects as per the randomly allocated sequence. They were instructed to take one capsule from each bottle daily morning with water after breakfast for a period of 4 weeks. Thereafter, at each visit, the subject was given a new sealed packet containing two sealed bottles and asked to consume in the same pattern as instructed earlier.

The pharmacist provided the subjects with bottles containing the study products, which were sequentially numbered. The bottles were dispensed as per the randomly allocated sequence. The principal investigator and the subjects were blinded. The allocations were unblinded after the completion of the study period for the purpose of the tabulation of data and statistical analysis.

2.5. Interventions. All the randomized subjects received the following interventions as per the groups allotted to them.

2.5.1. Group A. They take two soft gelatin capsules of Product 1 once a day, to provide a total dose of $2000 \mathrm{mg}$ of $\omega$-3FA, containing $600 \mathrm{mg}$ of EPA $+400 \mathrm{mg}$ of DHA.

2.5.2. Group B. They take one soft gelatin capsule of Product $1+$ One soft gelatin capsule of Product 2 once a day to provide a total dose of $2000 \mathrm{mg}$ of $\omega$-3FA, containing $600 \mathrm{mg}$ of EPA $+400 \mathrm{mg}$ of DHA and $10 \mathrm{mg}$ of PCC (equivalent to $200 \mathrm{mcg}$ of $\mathrm{Cr}^{3+}$ ).

2.5.3. Group C. They take two soft gelatin capsules of Product 2 once a day to provide a total of $2000 \mathrm{mg}$ of $\omega$-3FA, containing $600 \mathrm{mg}$ of EPA $+400 \mathrm{mg}$ of DHA and $20 \mathrm{mg}$ of PCC (equivalent to $400 \mathrm{mcg}$ of $\mathrm{Cr}^{3+}$ ). 


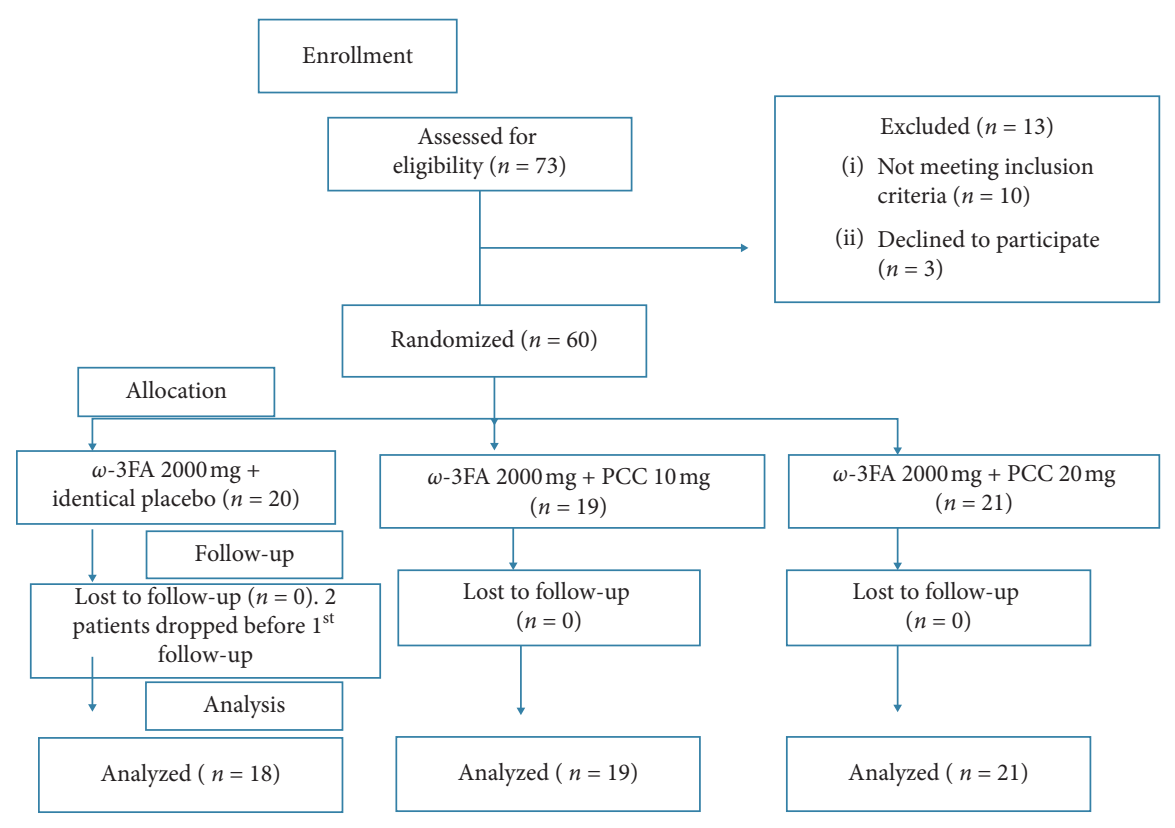

Figure 1: Consort flow diagram of the study.

2.6. The Efficacy Variables. The primary efficacy measures were improvement in endothelial function as assessed by more than $6 \%$ change in reflection index at 12 weeks in all the treatment groups, improvement in endothelial function and oxidative stress biomarkers (NO, GSH, and MDA), inflammation biomarker (hsCRP), vascular inflammation biomarkers (ET-1, ICAM-1, and VCAM-1), glycosylated haemoglobin $(\mathrm{HbA1c})$, platelet aggregation, and lipid profile. Secondary efficacy measure was the safety and tolerability assessment of the study products.

2.7. Assessment of Endothelial Function. Endothelial function was assessed using the salbutamol challenge test employing digital volume plethysmography, following the methods of Chowienczyk et al. [34] and Naidu et al., [35]. The patients were inspected at the supine position after resting for 5 minutes. A digital volume pulse (DVP) was recorded using a photo-plethysmograph (Pulse Trace PCA2, PT200, Micro Medical, Kent, UK) transmitting infrared light at $940 \mathrm{~nm}$, placed on the index finger of the right hand. The signal from the plethysmograph was digitized using a 12-bit analog to a digital converter with a sampling frequency of $100 \mathrm{~Hz}$. DVP waveforms were recorded over 20 -second period, and the height of the late systolic/early diastolic portion of the DVP was expressed as a percentage of the amplitude of the DVP to yield the reflection index (RI), following the procedure of Millasseau et al. [36]. After the DVP recording, three measurements of reflection index (RI) were calculated, and the mean value was determined. The patients were then administered with $400 \mu \mathrm{g}$ of salbutamol by inhalation. After 15 minutes, three more measurements of RI were recorded, and the difference in mean RI before and after administration of salbutamol was used for assessing endothelial function. A change in $\leq 6 \%$ of RI postsalbutamol administration was considered as endothelial dysfunction.
2.8. Biomarker Evaluation. Nitric oxide [37, 38] (by colorimetric detection with Griess reagents), MDA [39] (by thiobarbituric acid reactive substance test), and GSH (by Ellman's method) [40] levels were estimated spectrophotometrically. The ELISA method was employed to assess hsCRP, ET-1, ICAM-1, and VCAM-1 levels. Blood samples were collected at the department from the subjects after overnight fasting for the estimation of haemoglobin, blood urea and serum creatinine, liver function tests, and lipid profile, which included total cholesterol (TC), high-density lipoprotein cholesterol (HDL-C), low-density lipoprotein cholesterol (LDL-C), very low-density lipoprotein cholesterol (VLDL-C), and TGs, using appropriate standard techniques. A platelet aggregation test with $10 \mu \mathrm{M} / \mathrm{mL}$ ADP and $2 \mu \mathrm{g} / \mathrm{mL}$ collagen was performed by employing a platelet aggregometry test (Chrono-log light transmittance aggregometry).

Serum sample was used to estimate biomarkers (MDA, NO, GSH, hsCRP, ICAM-1, VCAM-1, and ET-1) and other biochemical tests. Whole blood samples were collected in EDTA tubes and were used for estimating HbA1c. The analysis of biomarkers (MDA, NO, GSH, hsCRP, ICAM-1, VCAM-1, and ET-1) was performed at the end of the study period in one batch. After collection of the blood samples, serum was separated and stored at -80 degrees centigrade. All the tests were performed at our institutional laboratory.

2.9. Follow-Up Visits. Salbutamol challenge test employing digital volume plethysmography was used to assess endothelial function as estimated by RI at every visit (baseline, 4, 8 , and 12 weeks). Blood samples were collected, after an overnight fast, for evaluation of MDA, NO, GSH, hsCRP, ICAM-1, VCAM-1, and ET-1, at baseline, 4, and 12 weeks. Lipid profile, $\mathrm{HbA1c}$, and platelet aggregation were evaluated at baseline and at the end of 12 weeks. 
2.10. Safety Evaluation. A complete physical examination was done at each visit. Safety laboratory investigations for hematological, hepatic, and renal biochemical parameters were evaluated at baseline and the end of the study, and if necessary, during the study. Subjects were enquired for the presence of adverse drug reactions (ADR) at every visit. ADRs were recorded in the case report form if any.

2.11. Compliance Verification. Pill-count method was used for the assessment of compliance with the study medications. Compliance was considered good if a patient received $>80 \%$, fair between $60 \%$ and $80 \%$, or poor if $<60 \%$ of the dispensed medication.

2.12. Sample Size Determination. A total sample size of 75 was estimated to be needed to enroll 66 subjects, assuming a pooled standard deviation of 2 units to achieve a power of $80 \%, 5 \%$ significance, screen failure $10 \%$, and a dropout rate of $10 \%$.

2.13. Statistical Analyses. Study data are expressed as mean \pm SD. "Paired $t$-test" was used to compare the change in biomarkers from baseline to post-treatment within groups, and ANOVA for between group comparisons. Post hoc analysis between the groups was done using Tukey's test. A $p$-value of less than 0.05 was considered statistically significant. All statistical analysis was performed using licensed version of the software GraphPad Prism8.

\section{Results}

From a total of 73 screened subjects, 60 eligible subjects were randomized in the study by computer generated block randomization and 58 completed 12 weeks of treatment. Two subjects dropped out of the study before the first followup. A total of 18 subjects in Group A, 19 in Group B, and 21 in Group C completed 12 weeks of treatment. Table 1 depicts the demographic characteristics of the three groups. There were no significant differences between treatment groups in baseline characteristics, including age and body mass index (BMI), indicating a homogenous population.

3.1. Endothelial Function (RI\%). Endothelial function was assessed by evaluating RI. As seen from Table 2, all the groups have shown significant improvement in RI, indicating an improvement in endothelial function at 12 $(p \leq 0.0001), 8(p \leq 0.0001)$ as well as $4(p \leq 0.05)$ weeks. Further analysis showed that treatment with Group $C$ was significantly better than Group B and Group A at 12 weeks $(p \leq 0.001)$.

3.2. Endothelial Marker. A statistically significant difference in mean \% change was seen in the levels of $\mathrm{NO}(+5.15$, $+10.11 \%$, $+25.14 \%$ ) as depicted in Table 3 .
3.3. Oxidative Stress Markers. The biomarkers of oxidative stress showed improvement at 12 weeks with all the groups. As depicted in Table 3, statistically significant difference in mean \% change was seen: MDA $(-0.74 \%,-6.74 \%,-18.46 \%)$, and GSH $(+1.01,+4.96 \%,+15.39 \%)$.

3.4. Inflammation Marker. The results were similar, as shown in Table 3 with the markers of inflammation at 12 weeks: hsCRP $(-1.39 \%,-5.03 \%,-21.56 \%)$, ICAM-1 (-8.84\%, $-16.24 \%,-23.57 \%)$, VCAM-1 (-3.58\%, $-17.58 \%$, $-31.62 \%)$, and ET-1 (-8.45\%, $-12.37 \%,-26.89 \%)$. Group B was better than Group A $(p<0.01)$, while Group C was more efficacious than the other two groups $(p<0.001)$. There was no significant difference between Groups A and B in improving the ET-1 levels.

3.5. Lipid Profiles. As shown in Table 4, at 12 weeks, statistical significance was seen with all the three groups in mean \% change of HDL $(1.43 \%, 4.98 \%, 9.55 \%)$, VLDL $(-2.28 \%,-7.30 \%,-13.15 \%)$, and TG $(-2.33 \%,-6.87 \%$, $-13.92 \%)$, while Groups B and C have also shown significant mean \% change in the levels of TC $(-3.48 \%,-6.94 \%)$ and LDL-C $(-5.12 \%,-10.98 \%)$. The significance with Group A was $p<0.05$, while Group B and Group C had shown a significance of $p<0.0001$ when compared to baseline. Between-group analysis showed Group $C$ to be better than Group A $(p<0.001)$, as well as Group B $(p<0.01)$.

3.6. Effect on Glycosylated Haemoglobin A1c (HbA1c \%). A statistically significant improvement $(p \leq 0.01)$ in HbA1c was seen with only Group C from 6.17 to 5.89 , which was a modest improvement.

3.7. Platelet Aggregation. No effect was recorded on platelet aggregation with any of the groups.

3.8. Safety Evaluation. All safety haematological and biochemical parameters were within normal limits with all the groups at the end of the treatment. Three subjects in Group $\mathrm{C}$ complained of gastrointestinal intolerance, and one patient reported nausea, while two reported dyspepsia, which subsided with symptomatic treatment. However, no subject in any of the groups discontinued the study due to adverse events.

\section{Discussion}

Significant, though modest, improvement in endothelial function, biomarkers of oxidative stress, systemic and vascular inflammation, and lipid profile was seen with $\omega$-3FA, but the addition of PCC, especially at $20 \mathrm{mg}$ dose $(400 \mathrm{mcg}$ of $\left.\mathrm{Cr}^{3+}\right)$, significantly increased the efficacy of $\omega$-3FA.

In the present study, RI was measured to assess endothelial function, using salbutamol challenge test employing digital volume plethysmography (DPG). This test is a simple, noninvasive, reproducible and a reliable 
TABLE 1: Baseline characteristics of subjects.

\begin{tabular}{|c|c|c|c|}
\hline Parameter & $\begin{array}{c}\omega-3 \text { FA } 2000 \mathrm{mg} \\
(\mathrm{A}) \\
(n=18) \\
\end{array}$ & $\begin{array}{c}\omega-3 \mathrm{FA} 2000 \mathrm{mg}+\text { PCC } 10 \mathrm{mg} \\
(\mathrm{B}) \\
(n=19) \\
\end{array}$ & $\begin{array}{c}\omega-3 \mathrm{FA} 2000 \underset{(\mathrm{C})}{\mathrm{mg}}+\text { PCC } 20 \mathrm{mg} \\
(n=21) \\
\end{array}$ \\
\hline Age in yrs & $50.89 \pm 4.34$ & $52.58 \pm 4.57$ & $54.33 \pm 6.41$ \\
\hline $\operatorname{Sex}(M / F)$ & $11 / 7$ & $12 / 7$ & $13 / 8$ \\
\hline Bodyweight (kg) & $79.78 \pm 3.57$ & $80.92 \pm 3.70$ & $80.08 \pm 4.41$ \\
\hline Height $(\mathrm{cm})$ & $161.0 \pm 4.84$ & $161.4 \pm 4.89$ & $160.9 \pm 5.20$ \\
\hline $\operatorname{BMI}\left(\mathrm{kg} / \mathrm{m}^{2}\right)$ & $30.78 \pm 0.79$ & $31.02 \pm 0.73$ & $30.94 \pm 0.71$ \\
\hline
\end{tabular}

Values expressed as mean \pm SD. SD: standard deviation. BMI: Body Mass Index.

method [35]. It does not require trained personnel, unlike flow-mediated vasodilation (FMD), which requires sophisticated instrument and a skilled operator and has a chance for large interindividual variability in the assessment of ED [41]. In a study done by Skulas Ray et al. [42] on subjects with moderate hypertriglyceridemia, two different doses of $\omega$-3FA (0.85 and $3.4 \mathrm{~g} /$ day) taken for eight weeks showed no effect on endothelial function. In their study, baseline endothelial function testing was absent. In another study by Wong et al. [43], 12-week therapy with $\omega$-3FA (4 g/day) in type $2 \mathrm{DM}$, no improvement in endothelial function was noted. In a previous study [28] done at our department, we have shown the beneficial effect of PCC on endothelial function in T2DM patients. In the present study, we have shown that the addition of PCC, especially at $20 \mathrm{mg}\left(400 \mathrm{mcg}\right.$ of $\left.\mathrm{Cr}^{3+}\right)$, significantly enhanced the efficacy of $\omega$-3FA in improving endothelial function in MetS subjects, unlike the study reported by Wong et al. We assume that highly significant improvement in endothelial function can be attributed to the combination of $\omega$-3FA with PCC.

Malondialdehyde (MDA) is a byproduct formed by lipid peroxidation due to free radical generation. We have shown a significant reduction in MDA with all the groups. The combination of $\omega-3 \mathrm{FA}+20 \mathrm{mg}$ of PCC had shown better results when compared to the other two groups. Our results are similar to those of Shifdar et al. [44], who have related the potential mechanism for the decrease in MDA to the assembly of omega-3 fatty acids in membrane lipids and lipoproteins making the double bonds less available for free radical attack, inhibition of the prooxidant enzyme phospholipase A2 and stimulation of antioxidant enzymes. So, we assume that combination of $\omega-3 F A$ with PCC seems to have a more potent effect than $\omega$-3FA alone.

In a 12-week study by Asemi et al. [45], $\omega$-3FA alone and its combination with alpha-tocopherol significantly increased NO levels with no change in GSH levels. They have attributed increased levels of NO to direct and indirect effects of $\omega$-3FA on the arterial wall. In our study, we reported highly significant improvement in $\mathrm{NO}$ and GSH levels at the end of 12 weeks with all the treatment groups, with Group C showing better results than the other two groups. The probable mechanism in improved
NO levels could be as mentioned above. The improvement in GSH levels could be attributed to the fact that $\omega$-3FA improves levels of glutathione reductase (GR) [46]. As previously stated, the highly significant improvement in Group B and Group C can be due to the combination of $\omega$-3FA with PCC, which seems to be more beneficial than $\omega$-3FA alone.

Yamada et al. [47] observed that EPA reduced the levels of both ICAM-1 and VCAM-1 after three months of treatment in metabolic syndrome subjects. These results are in agreement with the highly significant reduction in ICAM1, VCAM-1, and ET-1 levels in all the 3 treatment groups in the present study, with the best improvement in Group C. This effect may be attributed to the inhibitory effect of $\omega$-3FA on the endothelial expression of adhesion molecules [48]. The addition of PCC to $\omega$-3FA seems to have provided a highly significant response on biomarkers of vascular inflammation, when compared to $\omega$-3FA given alone.

In previous studies $[49,50]$ done at our department, we have shown the beneficial effects of PCC and its constituent materials on hsCRP. In a study by Schiano et al. [51], $\omega$-3FA did not show any effect on hsCRP levels. Similarly, Mackay et al. [52] reported no change in hsCRP and platelet aggregation with $\omega$-3FA. However, in the present study, we report significant changes in hsCRP levels, at 12-weeks, even with $\omega$-3FA alone (Group A), while Groups B and C had shown highly significant improvement. Such a result may be due to addition of PCC to $\omega$-3FA. We report no change in platelet aggregation, similar to the study by Mackay et al.

Koh et al. [53], in their study on subjects with hypertriglyceridemia, reported that $\omega$-3FA reduced TG levels. Similarly, JELIS study by Yokoyama [54] had reported improvement in TC and HDL levels. Two other studies by Cicerio and Colletti [55] in hypertriglyceridemia subjects and Sawada et al. [56] in subjects with impaired glucose metabolism and coronary artery disease reported improvement in TG, HDL-C, and LDL-C levels. They related this improvement in lipid parameters to improvement in endothelial dysfunction. Similar to these studies, we report significant improvement in all the parameters of lipid profile with $\omega-3$ FA + PCC (Groups B and C). Group A had improved the levels of HDL-C, VLDL-C, and TG extremely slightly. 


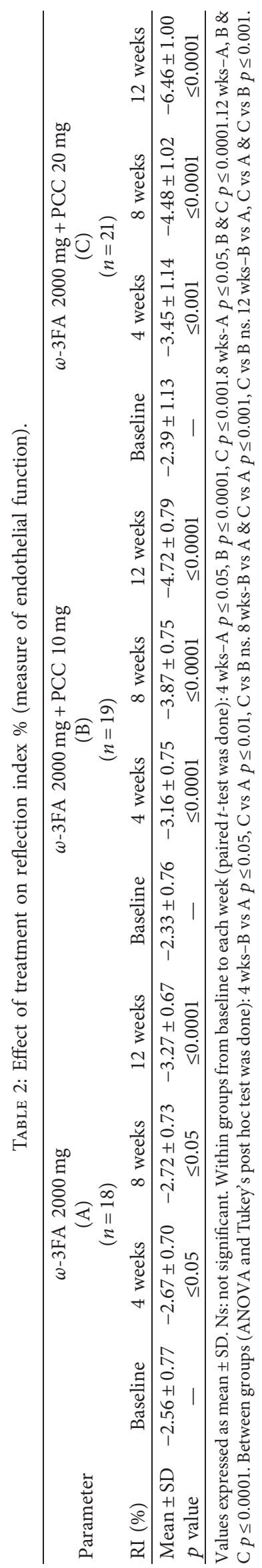




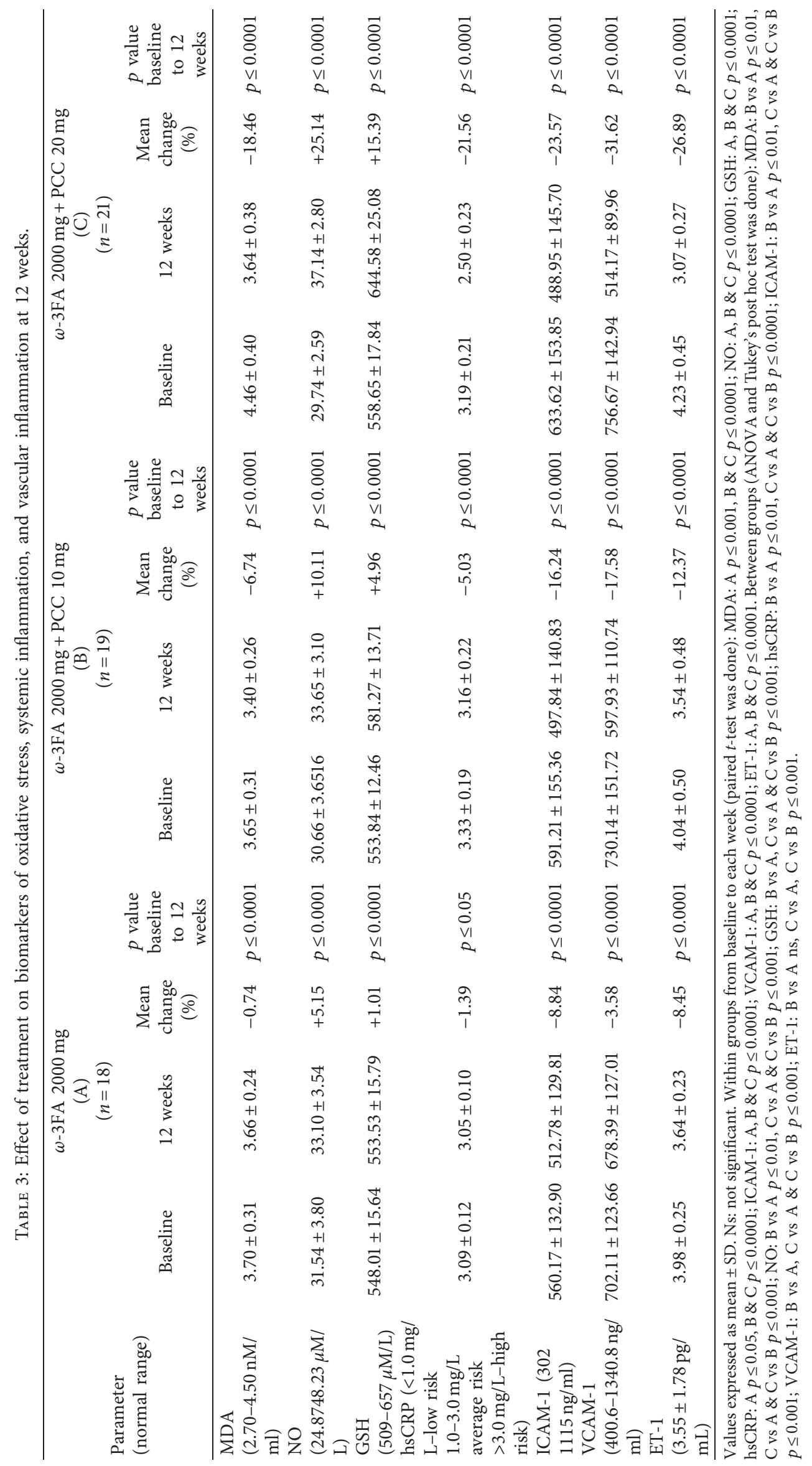




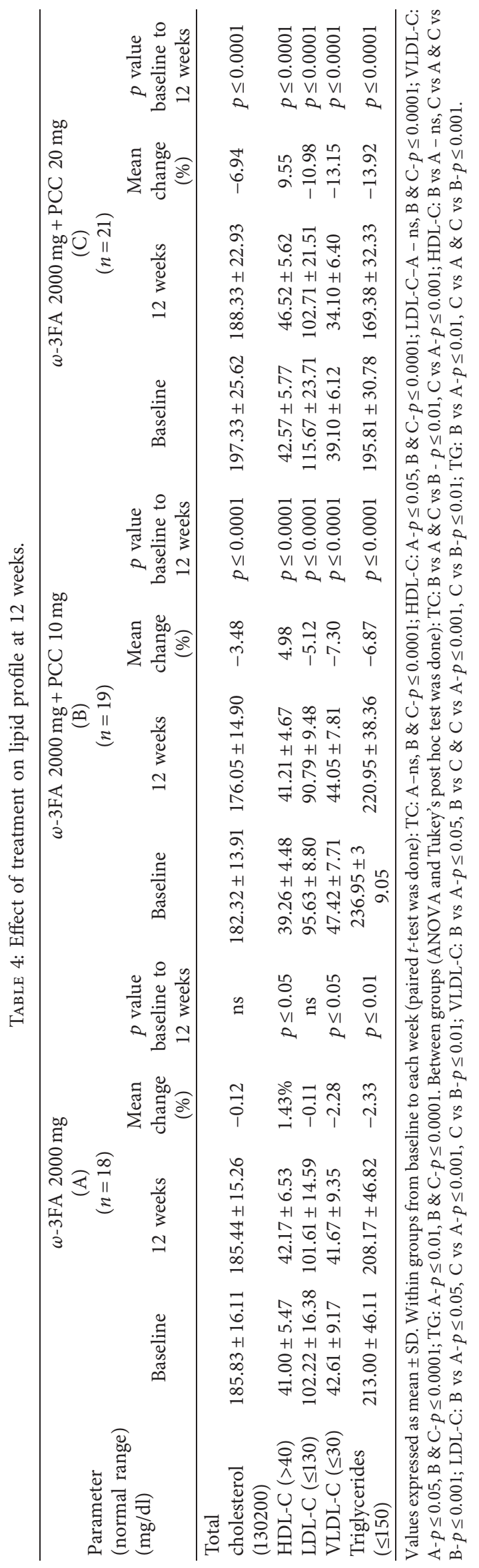




\section{Conclusion}

Omega-3 fatty acid ( $\omega$-3FA) alone and in combination with PCC $10 \mathrm{mg}\left(200 \mathrm{mcg} \mathrm{Cr}^{3+}\right)$ and $20 \mathrm{mg}\left(400 \mathrm{mcg} \mathrm{Cr}^{3+}\right)$ had a marked effect on endothelial function, accompanied by a significant improvement in the biomarkers NO, GSH, MDA, hsCRP, ICAM-1, VCAM-1, and ET-1, but the improvement was only modest with $\omega$-3FA alone, but highly significant with $\omega-3 F A$ and PCC combinations. There was a marked improvement in the lipid profile as well, and again the best improvement was seen with $\omega$-3FA + PCC $20 \mathrm{mg}(400 \mathrm{mcg}$ $\mathrm{Cr}^{3+}$ ), which is also the only group to show modest improvement in HbAlc. All the study medications were well tolerated, and no serious adverse events were observed. None of the subjects discontinued the study due to any adverse event suggesting the favorable safety profile of the treatments.

This study suggests that combining $\omega$-3FA with PCC may improve the cardiovascular morbidity in metabolic syndrome subjects in a dose-dependent manner. Further studies are warranted to substantiate the beneficial effect of this combination therapy when used as adjunctive therapy in subjects with metabolic syndrome. The limitation of this study is that it did not have a placebo group.

\section{Abbreviations}

ADR: Adverse drug reaction

ATP III: Adult treatment panel III

CRP: C-reactive protein

CV: Cardiovascular

CVD: Cardiovascular disease (CVD)

DM: Diabetes mellitus

GSH: Glutathione

HDL-C: High-density lipoprotein cholesterol

hsCRP: High sensitivity C-reactive protein

LDL-C: Low-density lipoprotein cholesterol

MDA: Malondialdehyde

MetS: Metabolic syndrome

NO: $\quad$ Nitric oxide

RI: $\quad$ Reflection index

ROS: Reactive oxygen species

TC: Total cholesterol (TC)

TG: $\quad$ Triglycerides

$\omega$-3FA: Omega-3 fatty acids.

\section{Data Availability}

The data will be handled on a case-by-case basis as per institutional policy.

\section{Conflicts of Interest}

The authors declare that there are no conflicts of interest regarding the publication of this paper.

\section{Acknowledgments}

The authors are grateful to Dr.Y.S.N. Raju, Professor of General Medicine, NIMS, for his clinical support; Dr. I. Sravanthi, Ayurvedic physician, for her expert advice; and study coordinator Mr. Muralidhar. The authors acknowledge Natreon, Inc., USA, for providing study medications and kits for biomarkers.

\section{References}

[1] S. M. Grundy, J. I. Cleeman, S. R. Daniels et al., "Diagnosis and management of the metabolic syndrome," Circulation, vol. 112, no. 17, pp. 2735-2752, 2005.

[2] P. W. F. Wilson, R. B. D'Agostino, H. Parise, L. Sullivan, and J. B. Meigs, "Metabolic syndrome as a precursor of cardiovascular disease and type 2 diabetes mellitus," Circulation, vol. 112, no. 20, pp. 3066-3072, 2005.

[3] U. Zafar, S. Khaliq, H. U. Ahmad, S. Manzoor, and K. P. Lone, "Metabolic syndrome: an update on diagnostic criteria, pathogenesis, and genetic links," Hormones, vol. 17, no. 3, pp. 299-313, 2018.

[4] K. G. M. M. Alberti, R. H. Eckel, S. M. Grundy et al., "Harmonizing the metabolic syndrome," Circulation, vol. 120, no. 16, pp. 1640-1645, 2009.

[5] K. G. M. Alberti, P. Zimmet, and J. Shaw, "The metabolic syndrome-a new worldwide definition," The Lancet, vol. 366, no. 9491, pp. 1059-1062, 2005.

[6] J. Olijhoek, Y. van der Graaf, J. D. Banga, A. Algra, T. J. Rabelink, and F. L. Visseren, "The Metabolic syndrome is associated with advanced vascular damage in patients with coronary heart disease, stroke, peripheral arterial disease or abdominal aortic aneurysm," European Heart Journal, vol. 25, no. 4, pp. 342-348, 2004.

[7] F. Guerrero-Romero, M. Rodríguez-Morán, and M. Hypomagnesemia, "Hypomagnesemia, oxidative stress, inflammation, and metabolic syndrome," Diabetes/Metabolism Research and Reviews, vol. 22, no. 6, pp. 471-476, 2006.

[8] N. Demircan, A. Gure, F. Armutcu, M. Unalacak, E. Aktunc, and H. Atmaca, "The evaluation of serum cystatin C, malondialdehyde, and total antioxidant status in subjects with metabolic syndrome," Medical Science Monitor, vol. 14, no. 2, pp. 97-101, 2008.

[9] J. E. Barbato, B. S. Zuckerbraun, M. Overhaus, K. G. Raman, and E. Tzeng, "Nitric oxide modulates vascular inflammation and intimal hyperplasia in insulin resistance and the metabolic syndrome," American Journal of Physiology-Heart and Circulatory Physiology, vol. 289, no. 1, pp. H228-H236, 2005.

[10] Y. X. Sun, S. J. Hu, X. H. Zhang, J. Sun, C. H. Zhu, and Z. J. Zhang, "Plasma levels of vWF and NO in subjects with metabolic syndrome and their relationship with metabolic disorders," Zhejiang Da Xue Xue Bao Yi Xue Ban, vol. 35, no. 3, pp. 315-318, 2006.

[11] N. Goutzourelas, M. Orfanou, I. Charizani, G. Leon, D. A. Spandidos, and D. Kouretas, "GSH levels affect weight loss in individuals with metabolic syndrome and obesity following dietary therapy," Experimental and Therapeutic Medicine, vol. 16, no. 2, pp. 635-642, 2018.

[12] O. Aouacheri, S. Saka, M. Krim, A. Messaadia, and I. Maidi, "The investigation of the oxidative stress-related parameters in type 2 diabetes mellitus," Canadian Journal of Diabetes, vol. 39, no. 1, pp. 44-49, 2015.

[13] G. Kressel, B. Trunz, A. Bub et al., "Systemic and vascular markers of inflammation in relation to metabolic syndrome and insulin resistance in adults with elevated atherosclerosis risk," Atherosclerosis, vol. 202, no. 1, pp. 263-271, 2009.

[14] I. Jialal, B. Adams-Huet, and S. Devaraj, "Monocyte cell adhesion molecule receptors in nascent metabolic syndrome," Clinical Biochemistry, vol. 49, no. 6, pp. 505-507, 2016. 
[15] J. E. Deanfield, J. P. Halcox, and T. J. Rabelink, "Endothelial function and dysfunction," Circulation, vol. 115, no. 10, pp. 1285-1295, 2007.

[16] G. Maulucci, F. Cipriani, D. Russo et al., "Improved endothelial function after short-term therapy with evolocumab," Journal of Clinical Lipidology, vol. 12, no. 3, pp. 669-673, 2018.

[17] A. P. Davel, Q. Lu, M. E. Moss et al., "Sex-specific mechanisms of resistance vessel endothelial dysfunction induced by cardiometabolic risk factors," Journal of the American Heart Association, vol. 7, no. 4, Article ID e007675, 2018.

[18] M. A. Creager, S. J. Gallagher, X. J. Girerd, S. M. Coleman, V. J. Dzau, and J. P. Cooke, "L-arginine improves endothelium-dependent vasodilation in hypercholesterolemic humans," Journal of Clinical Investigation, vol. 90, no. 4, pp. 1248-1253, 1992.

[19] J. D. Neaton, H. Blackburn, D. Jacobs et al., "Serum cholesterol level and mortality findings for men screened in the multiple risk factor intervention trial. Multiple risk factor intervention trial research group," Archives of Internal Medicine, vol. 152, no. 7, pp. 1490-1500, 1992.

[20] A. F. G. Cicero, M. Rosticci, M. Morbini et al., "Lipid-lowering and anti-inflammatory effects of omega 3 ethyl esters and krill oil: a randomized, cross-over, clinical trial," Archives of Medical Science, vol. 3, no. 3, pp. 507-512, 2016.

[21] D. L. Mendrick, A. M. Diehl, L. S. Topor et al., "Metabolic syndrome and associated diseases: from the bench to the clinic," Toxicological Sciences: An Official Journal of the Society of Toxicology, vol. 162, no. 1, pp. 36-42, 2018.

[22] S. Lim and R. H. Eckel, "Pharmacological treatment and therapeutic perspectives of metabolic syndrome," Reviews in Endocrine \& Metabolic Disorders, vol. 15, no. 4, pp. 329-341, 2014.

[23] M. Rameshrad, B. M. Razavi, G. A. A. Ferns, and H. Hosseinzadeh, "Pharmacology of dipeptidyl peptidase- 4 inhibitors and its use in the management of metabolic syndrome: a comprehensive review on drug repositioning," Daru: Journal of Faculty of Pharmacy, Tehran University of Medical Sciences, vol. 27, no. 1, pp. 341-360, 2019.

[24] J. Endo and M. Arita, "Cardioprotective mechanism of omega-3 polyunsaturated fatty acids," Journal of Cardiology, vol. 67 , no. 1, pp. 22-27, 2016.

[25] W. S. An, H. J. Kim, K.-H. Cho, and N. D. Vaziri, "Omega-3 fatty acid supplementation attenuates oxidative stress, inflammation, and tubulointerstitial fibrosis in the remnant kidney," American Journal of Physiology-Renal Physiology, vol. 297, no. 4, pp. F895-F903, 2009.

[26] A. Naqshbandi, S. Rizwan, M. Khan, and F. Khan, "Dietary flaxseed oil supplementation ameliorates the effect of cisplatin on brush border membrane enzymes and antioxidant system in rat intestine," Human \& Experimental Toxicology, vol. 32, no. 4, pp. 385-394, 2013.

[27] C. A. Fahs, H. Yan, S. Ranadive et al., "The effect of acute fishoil supplementation on endothelial function and arterial stiffness following a high-fat meal," Applied Physiology, Nutrition, and Metabolism, vol. 35, no. 3, pp. 294-302, 2010.

[28] P. Usharani, C. G. Devi, K. K. Kishore, and C. U. Kumar, "Effect of proprietary chromium complex and its individual components versus chromium picolinate, chromium polynicotinate and chromium dinicocysteinate on endothelial function, biomarkers and lipid profile in type 2 diabetics-a randomized, double-blind, placebo-controlled study," International Journal of Pharmaceutical Sciences and Research, vol. 8, no. 5, pp. 2267-2276, 2017.
[29] A. Das, M. S. El Masry, S. C. Gnyawali et al., "Skin transcriptome of middle-aged women supplemented with natural herbo-mineral shilajit shows induction of microvascular and extracellular matrix mechanisms," Journal of the American College of Nutrition, vol. 38, no. 6, pp. 526-536, 2019.

[30] S. Joukar, H. Najafipour, S. Dabiri, M. Sheibani, and N. Sharokhi, "Cardioprotective effect of mumie (shilajit) on experimentally induced myocardial injury," Cardiovascular Toxicology, vol. 14, no. 3, pp. 214-221, 2014.

[31] U. Pingali, C. Nutalapati, and V. S. Illendulla, "Evaluation of the effect of fish oil alone and in combination with a proprietary chromium complex on endothelial dysfunction, systemic inflammation and lipid profile in type 2 diabetes mellitus-a randomized, double-blind, placebo-controlled clinical study," Diabetes, Metabolic Syndrome and Obesity: Targets and Therapy, vol. 13, pp. 31-42, 2020.

[32] International Diabetes Federation, "The IDF consensus worldwide definition of the metabolic syndrome," 2017, https://www.idf.org/e-library/consensus-statements/60idfconsensus-worldwide-definitionof-the-metabolicsyndrome.html.

[33] World Medical Association, "World medical association declaration of Helsinki," JAMA, vol. 310, no. 20, pp. 2191-2194, 2013.

[34] P. J. Chowienezyk, R. P. Kelly, H. MacCallum, S. C. Millasseau, T. L. G. Anderson, and R. G. Gosling, "Photoplethysmographic assessment of pulse wave reflection: blunted response to endothelium dependent beta2-adrenergic vasodilation in type II diabetes mellitus," Journal of American College of Cardiology, vol. 34, no. 7, pp. 2007-2014, 1999.

[35] M. R. Naidu, Y. Sridhar, P. Rani, and A. Mateen, "Comparison of two $\beta 2$ adrenoceptor agonists by different routes of administration to assess human endothelial function," Indian Journal of Pharmacology, vol. 39, no. 3, pp. 168-169, 2007.

[36] S. C. Millasseau, R. P. Kelly, J. M. Ritter, and P. J. Chowienczyk, "Determination of age-related increases in large artery stiffness by digital pulse contour analysis," Clinical Science, vol. 103, no. 4, pp. 371-377, 2002.

[37] D. Yao, A. G. Vlessidis, and N. P. Evmiridis, "Determination of nitric oxide in biological samples," Microchimica Acta, vol. 147, pp. 1-20, 2004.

[38] S. Verma, M. R. Buchanan, and T. J. Anderson, "Endothelial function testing as a biomarker of vascular disease," Circulation, vol. 108, no. 17, pp. 2054-2059, 2003.

[39] J. Vidyasagar, N. Karunakar, M. S. Reddy, K. Rajnarayana, T. Surender, and D. R. Krishna, "Oxidative stress and antioxidant status in acute organophosphorous insecticide poisoning," Indian Journal of Pharmacology, vol. 36, pp. 76-79, 2004.

[40] G. L. Ellman, “Tissue sulfhydryl groups," Archives of Biochemistry and Biophysics, vol. 82, no. 1, pp. 70-77, 1959.

[41] C. Rambaran, B. Jiang, J. M. Ritter, A. Shah, L. Kalra, and P. J. Chowienczyk, "Assessment of endothelial function: comparison of the pulse wave response to $\beta 2$-adrenoceptor stimulation with flow mediated dilatation," British Journal of Clinical Pharmacology, vol. 65, no. 2, pp. 238-243, 2008.

[42] A. C. Skulas-Ray, P. M. Kris-Etherton, W. S. Harris, J. P. Vanden Heuvel, P. R. Wagner, and S. G. West, "Doseresponse effects of omega-3 fatty acids on triglycerides, inflammation, and endothelial function in healthy persons with moderate hypertriglyceridemia," The American Journal of Clinical Nutrition, vol. 93, no. 2, pp. 243-252, 2011.

[43] C.-Y. Wong, K.-H. Yiu, S.-W. Li et al., "Fish-oil supplement has neutral effects on vascular and metabolic function but 
improves renal function in patients with type 2 diabetes mellitus," Diabetic Medicine, vol. 27, no. 1, pp. 54-60, 2010.

[44] F. Shidfar, A. Keshavarz, S. Hosseyni, A. Ameri, and S. Yarahmadi, "Effects of omega-3 fatty acid supplements on serum lipids, apolipoproteins and malondialdehyde in type 2 diabetes subjects," Eastern Mediterranean Health Journal, vol. 14, no. 2, pp. 305-313, 2008.

[45] Z. Asemi, A. Soleimani, H. Shakeri, N. Mazroii, and A. Esmaillzadeh, "Effects of omega-3 fatty acid plus alphatocopherol supplementation on malnutrition-inflammation score, biomarkers of inflammation and oxidative stress in chronic hemodialysis patients," International Urology and Nephrology, vol. 48, no. 11, pp. 1887-1895, 2016.

[46] T. E. Sorto-Gomez, G. G. Ortiz, F. P. Pacheco-Moises et al., "Effect of fish oil on glutathione redox system in multiple sclerosis," American Journal of Neurodegenerative Diseases, vol. 5, no. 2, pp. 145-151, 2016.

[47] H. Yamada, M. Yoshida, Y. Nakano et al., "In vivo and in vitro inhibition of monocyte adhesion to endothelial cells and endothelial adhesion molecules by eicosapentaenoic acid," Arteriosclerosis, Thrombosis, and Vascular Biology, vol. 28, no. 12, pp. 2173-2179, 2008.

[48] P. C. Calder, "Omega-3 polyunsaturated fatty acids and inflammatory processes: nutrition or pharmacology?" British Journal of Clinical Pharmacology, vol. 75, no. 3, pp. 645-662, 2013.

[49] P. Usharani, N. Fatima, and N. Muralidhar, "Effects of Phyllanthus emblica extract on endothelial dysfunction and biomarkers of oxidative stress in subjects with type 2 diabetes mellitus: a randomized, double-blind, controlled study," Diabetes, Metabolic Syndrome and Obesity: Targets and Therapy, vol. 6, pp. 275-284, 2013.

[50] P. Usharani, P. V. Kishan, N. Fatima, and U. C. Kumar, "A comparative study to evaluate the effect of highly standardised aqueous extracts of Phyllanthus emblica, withania somnifera and their combination on endothelial dysfunction and biomarkers in subjects with type II diabetes mellitus," International Journal of Pharmaceutical Sciences and Research, vol. 5, no. 7, pp. 2687-2697, 2014.

[51] V. Schiano, E. Laurenzano, G. Brevetti et al., "Omega-3 polyunsaturated fatty acid in peripheral arterial disease: effect on lipid pattern, disease severity, inflammation profile, and endothelial function," Clinical Nutrition, vol. 27, no. 2, pp. 241-247, 2008.

[52] I. Mackay, I. Ford, F. Thies, S. Fielding, P. Bachoo, and J. Brittenden, "Effect of omega-3 fatty acid supplementation on markers of platelet and endothelial function in patients with peripheral arterial disease," Atherosclerosis, vol. 221, no. 2, pp. 514-520, 2012.

[53] K. K. Koh, M. J. Quon, R. S. Rosenson, W. J. Chung, and S. H. Han, "Vascular and metabolic effects of treatment of combined hyperlipidemia: focus on statins and fibrates," International Journal of Cardiology, vol. 124, no. 2, pp. 149159, 2007.

[54] M. Yokoyama, "Japan EPA Lipid Intervention Study (JELIS). Randomized clinical trial involving primary and secondary prevention of cardiovascular events with EPA in hypercholesterolemia," Nippon Ronen Igakkai Zasshi, vol. 46, no. 1, pp. 22-25, 2009.

[55] A. F. Cicero and A. Colletti, "Role of phytochemicals in the management of metabolic syndrome," Phytomedicine: International Journal of Phytotherapy and Phytopharmacology, vol. 23, no. 11, pp. 1134-44, 2016.
[56] T. Sawada, H. Tsubata, N. Hashimoto et al., "Effects of 6month eicosapentaenoic acid treatment on postprandial hyperglycemia, hyperlipidemia, insulin secretion ability, and concomitant endothelial dysfunction among newly diagnosed impaired glucose metabolism subjects with coronary artery disease. An open label, single blinded, prospective randomized controlled trial," Cardiovascular Diabetology, vol. 15, no. 1, p. 21, 2016. 